\title{
Medication Safety Group clinician updates: a novel teaching programme to bridge the gap between the prescriber and the trust
}

\author{
Authors: Charlotte Strzelecki and Roya Behrouzi
}

\begin{abstract}
Aims
This project aimed to develop, implement and evaluate a novel medication safety teaching programme focused on updating foundation doctors about local medication-related patient safety issues and engaging them in trust-wide medication safety initiatives.
\end{abstract}

\section{Methods}

The Medication Safety Group (MSG) is a multidisciplinary panel responsible for comprehensively assessing and managing all medication-related factors associated with patient safety risks within our major acute hospital trust. Historically, dissemination of information from the MSG has been via ad hoc trust-wide emails or intranet alerts, and a significant disconnect existed between the MSG and prescribers; particularly junior doctors. Over 6 months, a novel medication safety teaching programme entitled the 'MSG clinician updates' has been developed to formally disseminate key areas of concern identified by the MSG directly to foundation doctors. Sessions have been delivered by two junior clinical MSG representatives in bimonthly 1-hour lectures as part of the compulsory foundation teaching programme. Sessions were delivered in parallel with bimonthly MSG meetings to ensure timely communication of hospital incident report trends, lessons learnt from medication related serious untoward incidents, national safety alerts and changes to trust medication policies.

\section{Results}

Evaluation of written feedback revealed high levels of satisfaction with MSG clinician updates. When asked to rate each session from 1-10 (10 = excellent, 1 = poor) for relevance, information provision and use of clinical examples, doctors gave mean scores of 8.7, 9.3 and 9.5 , respectively $(n=53)$. Case-based discussions were reported to be of significant benefit and review of the trust's medication guidelines improved trainees' knowledge of local resources. Some doctors felt feedback regarding prescribing errors often did not reach rotating doctors and these sessions helped close the feedback loop. Where specific local medication safety trends were identified, for example missed doses, doctors were encouraged to discuss and reflect upon how their practice could be improved, thus promoting real-time engagement in wider trust strategies to address medication related patient safety risks.

\section{Conclusion}

MSG clinician updates is a novel teaching programme that is improving dissemination of medication safety issues to foundation doctors by forming a direct link between prescribers and the MSG. The sessions have also provided a valuable forum for trainees to relay concerns back to the MSG. This has generated prescriber-led initiatives that were addressed by the MSG and actively engaged clinicians in medication safety improvements within the trust. This project is being expanded to widen access to all grades of clinician through incorporation into the unscheduled care directorate departmental teaching programme.

\section{Conflict of interest statement}

There are no conflicts of interest declared.

Authors: Manchester University NHS Foundation Trust, Manchester, UK 